\title{
Mitochondrial Quality Control and Parkinson's Disease: A Pathway Unfolds
}

\author{
Inês Pimenta de Castro • L. Miguel Martins • \\ Samantha Hui Yong Loh
}

Received: 7 September 2010 / Accepted: 4 November 2010 /Published online: 1 December 2010

(C) The Author(s) 2010. This article is published with open access at Springerlink.com

\begin{abstract}
Recent findings from genetic studies suggest that defective mitochondrial quality control may play an important role in the development of Parkinson's disease (PD). Such defects may result in the impairment of neuronal mitochondria, which leads to both synaptic dysfunction and cell death and results in neurodegeneration. Here, we review state-of-the-art knowledge of how pathways affecting mitochondrial quality control might contribute to $\mathrm{PD}$, with a particular emphasis on the molecular mechanisms employed by PTEN-induced putative kinase 1 (PINK1), HtrA2 and Parkin to regulate mitochondrial quality control.
\end{abstract}

Keywords Mitochondria $\cdot \mathrm{PINK} 1 \cdot \mathrm{HtrA} 2 \cdot$ Parkin .

Parkinson's disease $\cdot$ Unfolded proteins

\section{Introduction}

Parkinson's disease (PD) is a neurodegenerative disorder of the central nervous system that belongs to a group of conditions referred to as movement disorders. Efforts to understand the genetic basis of this disease have led to the identification of a number of gene mutations accounting for a small proportion of PD cases. Although the role of mitochondria in PD has been debated inconclusively in the past few decades, it was the identification of PD-causative

I. P. de Castro • L. M. Martins $(\bowtie) \cdot$ S. H. Y. Loh

Cell Death Regulation Laboratory, MRC Toxicology Unit,

Hodgkin Building, Lancaster Road,

Leicester LE1 9HN, UK

e-mail: martins.lmiguel@gmail.com

I. P. de Castro

IPATIMUP and Faculty of Pharmacy, University of Porto,

Porto, Portugal mutations in the mitochondrial putative protein kinase PINK1 that helped reignite the interest in the potential role of this organelle in the pathophysiology of familial PD. It is now becoming clear that PINKI acts in a mitochondrial quality control pathway and that other genes implicated in this disease are under its control. This suggests that a subset of genes involved in PD converge in a common pathway that modulates the fidelity of mitochondria. Given that most PD cases are sporadic with no known specific cause, this raises the interesting hypothesis that impaired mitochondrial quality control might be a defining causative feature of this disease. The purpose of this review is to summarise some of the recent molecular determinants of mitochondrial quality control regulated by PINK1.

\section{Mitochondria: A Force for Life and Death}

Midichlorians are microorganisms in the fictional Star Wars galaxy. These microscopic life forms reside within cells of all living things and communicate using the Force. The Force is an energy field created by all living things and harvested by the Jedi knights to protect the galaxy. The Force, however, has a 'Dark Side', and it can therefore be employed to cause death and destruction. Midichlorians not only help to communicate the Force, but they are also symbionts with all living things. Without them, life could not exist. The creator of Star Wars, George Lucas, stated that the midichlorians are based on the endosymbiotic theory, which explores the origin of mitochondria and plastids present in eukaryotic cells. In fact, midichlorians are loosely based on mitochondria, an organelle that sustains life in eukaryotic cells of multicellular organisms; however, these organelles are also known to bring about death and destruction within cells. 
It is widely accepted that the acquisition of mitochondria is a crucial event in the evolution of the eukaryotic cell [1]. According to the endosymbiotic theory, mitochondria evolved from a metabolically driven symbiotic relationship between aerobic bacteria and a nucleus-containing cell. As descendants of the original symbiont, mitochondria are organelles highly efficient in their ability to utilise molecular oxygen $\left(\mathrm{O}_{2}\right)$ and organic substrates, such as glucose and pyruvate, and to produce cellular energy in the form of ATP. The molecular machinery present in mitochondria for energy production is collectively referred to as the electron transport chain (ETC). The ETC consists of four protein complexes. Three of the complexes (I, III and IV) pump protons $\left(\mathrm{H}^{+}\right)$outwardly across the inner membrane to establish a gradient necessary for the production of ATP by a molecular motor (complex $\mathrm{V}$ or ATP synthase). During electron transport, $\mathrm{O}_{2}$ is converted to $\mathrm{H}_{2} \mathrm{O}$, and, particularly at complexes I and II, the free radical superoxide $\left(\mathrm{O}_{2}{ }^{-}\right)$is also generated as a by-product. Two important cofactors that modulate energy and free radical production are coenzyme $\mathrm{Q}_{10}$ at complex II and cytochrome c at complex IV [2]. It is noteworthy that excessive production of superoxide can enhance oxidative stress in dopaminergic neurons, eventually contributing to the demise of these cells in PD [3].

Mitochondria play a key role in eukaryotic cells by supplying the majority of the cellular energy in the form of ATP. However, a cell death-related role for this organelle is also now clearly established. When eukaryotic cells in multicellular organisms become worn out or damaged, they undergo cellular suicide (i.e. apoptosis). During this process, the cell blebs, shrinks and is packaged for recycling. When this process lacks proper control, either decreased or increased cell loss can occur, leading to diseases such as cancer or neurodegeneration. Apoptosis is actively controlled by mitochondria. When the mitochondrial pathway of apoptosis is activated, these organelles release several proteins that activate several proteases and other hydrolytic enzymes, leading to proteolysis, DNA fragmentation and chromatin condensation. The main mitochondrial effectors of apoptosis are proteins that paradoxically play important survival roles in nonapoptotic cells. These effectors include cytochrome c, which, in normal settings, is a component of the ETC, and the serine protease HtrA2 (also known as Omi) which seems to play a protective role during stress in the mitochondria of healthy cells [4]. The mitochondrial pathway of apoptosis is regulated by the BCL-2 family proteins (reviewed in Youle and Strasser [5]) and involves the activation of BCL-2 family members, such as BAX and BAK, which act by forming pores in the outer mitochondrial membrane (OMM) and allow the release of several pro-apoptotic proteins from the intermembrane space into the cytosol. The role of enhanced apoptosis as a causative mechanism for the death of dopaminergic neurons in PD patients is still controversial [6]. However, given that healthy mitochondria contain a multitude of molecules that, when released into the cytosol, lead to apoptotic cell death, it is reasonable to assume that the compromise of mitochondrial function in dopaminergic neurons contributes to their demise through the engagement of this form of cell suicide.

\section{Totally Addicted to Mitochondria}

In humans, most of the energy supplying the brain is generated by mitochondria. The majority of this energy is used for reversing the movement of ions that bring about action potentials and synaptic transmission in neurons. Neurons have very low glycolytic rates (reviewed in Bolanos and Almeida [7]) and therefore require mitochondria for energy production. Mitochondria also sequester and buffer cytoplasmic calcium; thus, their localisation plays a role in local regulation of intracellular calcium dynamics. Neurons are large, specialised cells, and the biogenesis of mitochondria in neurons takes place in the soma. This means that mitochondria normally have to travel large distances to deliver energy. Impressively, these organelles will travel up to $1 \mathrm{~m}$ in human motor neurons and, taking into account their maximum travelling speed, will take a minimum of 8 days to reach their destination. The majority of long-distance mitochondrial transport is mediated by motor proteins powered by ATP hydrolysis that shuttle mitochondria along microtubules (reviewed in MacAskill and Kittler [8]). Such mitochondrial transport is bidirectional, as damaged mitochondria may need to travel back to the cell soma for autophagic degradation. This degradation is mediated through autophagy, a molecular recycling programme for impaired and defective cellular components.

In neurons, the synaptic terminals are sites of high energy demand. Therefore, these structures rely heavily on mitochondria for energy production. Synaptic transmission requires high levels of cellular ATP for numerous energyconsuming processes, including the maintenance of synaptic membrane potential and reloading of synaptic vesicles with neurotransmitters. In addition, synaptic terminals may require mitochondria for efficient sequestration of calcium ions $\left(\mathrm{Ca}^{2+}\right)$ that are released into the cytosol to elicit the fusion of synaptic vesicles with the plasma membrane (reviewed in Ly and Verstreken [9]). Adult dopaminergic neurons of the substantia nigra pars compacta $(\mathrm{SNc})$ that degenerate in PD are known to generate action potentials in the absence of any synaptic input. The mechanism behind this autonomous firing capacity involves ion channels that enable the entry of $\mathrm{Ca}^{2+}$ into the cytosol (reviewed by 
Chan, Gertler and Surmeier [10]). This $\mathrm{Ca}^{2+}$ is actively removed by active pumping across the plasma membrane or sequestration in the lumen of the endoplasmic reticulum (ER) or the mitochondrial matrix, a process that requires energy in the form of ATP. Also, active $\mathrm{Ca}^{2+}$ buffering by mitochondria is likely to have a significant effect in the physiology of this organelle. It is therefore attractive to propose that the premature death of dopaminergic neurons in the SNc might be precipitated by a failure to control calcium homeostasis linked to a compromise of mitochondrial function.

As mentioned above, neurons are specialised cells that have foregone the capacity to undergo cell division in the adult brain. Mitochondria are essential for energy generation in neurons. As previously stated, however, mitochondria can also bring about cellular demise through apoptosis. It is therefore essential to keep the destructive power of this organelle under strict control. The release of apoptotic machinery from mitochondria in neuronal cells can have serious consequences. It is thought to play a role in the progression of neurodegenerative disease in humans. Thus, it is conceivable that mitochondrial dysfunction may, at an early stage, trigger deficits in neurons prior to synapse formation, resulting in loss of connectivity, whereas overwhelming damage might induce apoptosis and cause neuronal death (Fig. 1).

\section{Parkinson's Disease: Poisons to Genes}

Parkinson's disease (PD) is a common neurodegenerative disease characterised initially by the loss of dopaminergic neurons in the substantia nigra and later by a more widespread loss of other neurons in the brain. Most PD cases occur sporadically (i.e. they are of unknown cause). Between $10 \%$ and $15 \%$ of PD patients, however, have a family history of the disease, indicating that there is a strong genetic basis for this disease in this subgroup. The molecular pathogenesis of sporadic PD and the basis for selective dopaminergic neuronal loss remains unknown, and it is unclear whether gene mutations are involved in the development of this disease in this subgroup of patients. Epidemiological studies consistently link exposure to pesticides to a higher incidence of PD. In particular, pesticides such as rotenone and paraquat have been shown to cause PD-like conditions in rodent models. In addition, the mitochondrial toxin 1-methyl-4-phenyl-1,2,3,6-tetrahydropyridine (MPTP) was shown to be responsible for the onset of severe PD-like symptoms in a group of young drug users in the 1980s. It is clear that such drugs affect the ETC in mitochondria, resulting in loss of ATP and perhaps an increase in free-radical generation. This evidence suggests that exposure to mitochondria-damaging agents might be

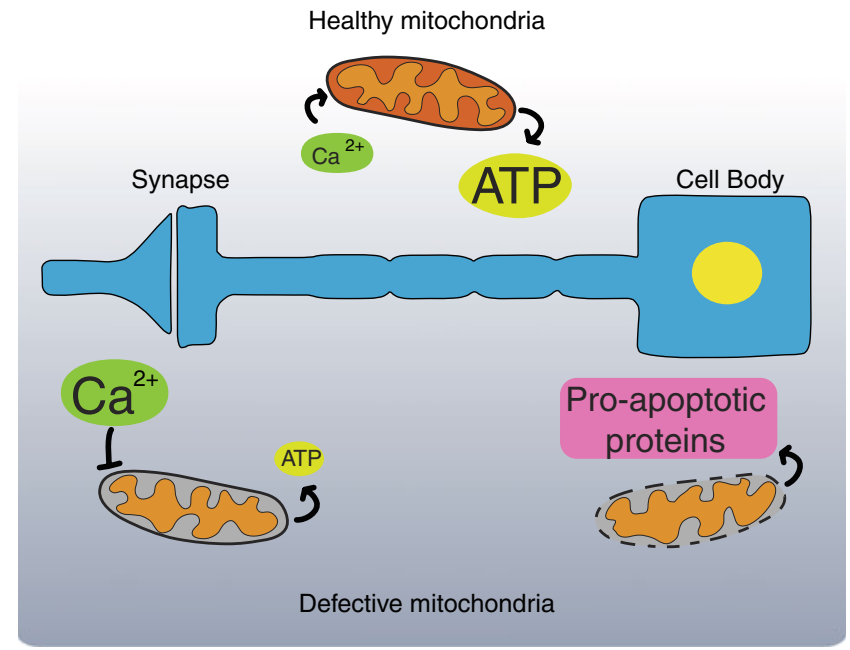

Fig. 1 Consequences of mitochondrial dysfunction in neurons. Neurons are large, specialised cells that rely on mitochondria for the majority of their energetic needs. A healthy mitochondrial population ensures the delivery of ATP as a source of energy and acts as a calcium buffering system, decreasing the concentration of this ion in the cytosol. At synapses, defective mitochondria are likely to be less capable of producing ATP and buffering calcium, possibly affecting efficient synaptic transmission. If these mitochondrial defects go unchecked, mitochondria will ultimately release pro-apoptotic factors, and, if this process occurs in the vicinity of the cell body, it is likely to result in neuronal death through apoptosis

important in the aetiology of PD in the sporadic patient population. Even though mitochondrial dysfunction has been inconclusively linked to PD in the past few decades, genetic evidence indicating mitochondrial involvement in this disease was obtained only recently. A major breakthrough occurred in a recent study in which researchers identified disease-causing mutations in PTEN-induced putative kinase 1 (PINK1) in familial PD [11]. More recently, mutations in the mitochondrial serine protease $\mathrm{HtrA} 2$ were reported to be associated with PD [12]. The role of HtrA2 in familial PD, however, remains controversial.

\section{PINK1: Lessons from Imperfect Models}

PINK1 is a putative mitochondrial kinase that acts to protect neurons from oxidative stress. In addition, it controls mitochondrial morphology in mammals and insects (reviewed in Thomas et al. [13]). Although animal models of PD fail to recapitulate all of the features of PD pathology in humans [14], they have proven to be invaluable tools for elucidating some of the mechanisms through which PINK1 affects mitochondrial function. Although the currently accepted models suggest that PINK1 is a kinase, its direct substrates have so far remained elusive. Perhaps the best evidence to indicate that the kinase activity of PINK1 is required for its protective function comes from studies from 
Drosophila melanogaster. In these studies, it was shown that a kinase-inactive version of Pink1 failed to rescue the phenotypical features of pink1 loss-of-function flies [15]. The fruit fly has proven to be an important tool in the dissection of the molecular determinants of pinkl function. These studies, however, have presented conflicting data regarding the importance of this gene for the survival of dopaminergic neurons (see [16, 17]).

Recently, it was reported that a loss of PINK1 in mice leads to a significant decrease in dopamine release and a subsequent impairment of striatal synaptic plasticity, even in the absence of dopaminergic neuronal degeneration [18]. In Drosophila neurons, Pink1 deficiency also seems to affect synaptic function. Loss of Pink1 affects mitochondrial function and leads to a decrease in the ATP levels that prevents synaptic vesicles from being mobilised during rapid stimulation [19]. In addition, loss of PINK1 in mice may also affect synaptic activity due to defects in mitochondrial calcium handling. Abramov and colleagues proposed that PINK1 regulates calcium efflux from mitochondria via the mitochondrial $\mathrm{Na}^{+} / \mathrm{Ca}^{2+}$ exchanger. Deregulation of mitochondrial calcium handling can result in mitochondrial calcium overload that affects synaptic function. Such an overload leads to increased levels of reactive oxygen species (ROS), which can then lead to mitochondria-dependent apoptosis [20]. Altogether, despite the limitations of PD models in non-primate animal species, both mice and flies have provided us with valuable clues regarding the mechanisms through which PINK1 regulates mitochondrial function.

\section{Neuronal Mitochondria, the Good, the Bad and the Ugly}

Neurons require mitochondria to function, and in the past few years, numerous findings have suggested that disruptions of mitochondrial function and dynamics contribute to ageing and neurodegenerative diseases (recently reviewed in de Castro et al. [21]). The emerging view is that eukaryotic cells have developed exquisite mechanisms to cope with the diverse challenges imposed on mitochondrial integrity (Fig. 2). Mitochondria are therefore thought to have at least two levels of defence mechanisms that ensure their integrity and viability in individual cells (reviewed in Tatsuta and Langer [22]). The first line of defence is comprised of highly specific molecular quality control machinery composed of molecular chaperones and energy-dependent proteases that monitor the folding and assembly of mitochondrial proteins. In addition, these molecules prevent the accumulation of damaged proteins from mitochondria. This mitochondrial stress signalling results in the selective transcriptional upregulation of nuclear genes encoding for mitochondrial
Hsp70 and Hsp60 in cells experiencing elevated mitochondrial protein-conformational stress, therefore suggesting the existence of an unfolded protein response of mitochondrial origin $\left(\mathrm{UPR}^{\mathrm{mt}}\right)$. One of the major upstream components of this signalling pathway in the worm Caenorhabditis elegans has been recently found to be the orthologue of Escherichia coli ClpP, a molecule that functions in the bacterial heatshock response (reviewed in Broadley and Hartl [23]). These interesting observations suggest that mitochondria, as descendants of the original endosymbiont, have co-opted certain molecules of bacterial origin to stress signalling functions.

Once molecular quality control is overwhelmed within mitochondria, a second line of defence, termed organellar quality control, is thought to take over. This mechanism relies on the dynamic nature of mitochondrial populations to ensure the disposal of defective components. Mitochondrial dynamics are thought to be important in the control of mitochondrial turnover and bioenergetic efficiency. The combined functions of fusion, fission and autophagy are now emerging as essential organellar quality control mechanisms that promote the sequestration, sorting and elimination of functionally impaired mitochondria [24]. Finally, if both of these lines of defence fail, severe mitochondrial damage unleashes the apoptosis pathway that culminates with cell death.

Is it possible that the demise of dopaminergic neurons in PD results from a failure of either one of the two described quality control mechanisms in mitochondria? A positive answer to this question is starting to emerge from recent data derived from genetic studies in model organisms.

xThe first link between PD and mitochondrial molecular quality control was suggested by the finding of heterozygous missense mutations in HtrA2 in sporadic cases of PD [12]. The serine protease HtrA2 was initially identified as a mammalian homologue of the $E$. coli proteases HtrA/DegP and DegS [25]. The structural and biochemical similarity of mammalian HtrA2 to the bacterial DegS protease is particularly striking [26-28]. In Gram-negative bacteria, the DegS protease is involved in a signal transduction pathway that senses and responds to envelope stress (reviewed in Ruiz and Silhavy [29]). In mammalian cells, HtrA2 normally resides in the mitochondrial intermembrane space. Given the similarities between DegS and HtrA2 and taking into account that bacteria are the most likely ancestors of mitochondria, it is possible that HtrA2 evolved from DegS and now controls the levels of unfolded proteins in mitochondria.

The characterisation of mice lacking HtrA2 showed that loss of this protease results in a neurodegenerative phenotype with parkinsonian features. This phenomenon, however, is not due to the loss of dopaminergic neurons; it is instead caused by the loss of a subpopulation of striatal 


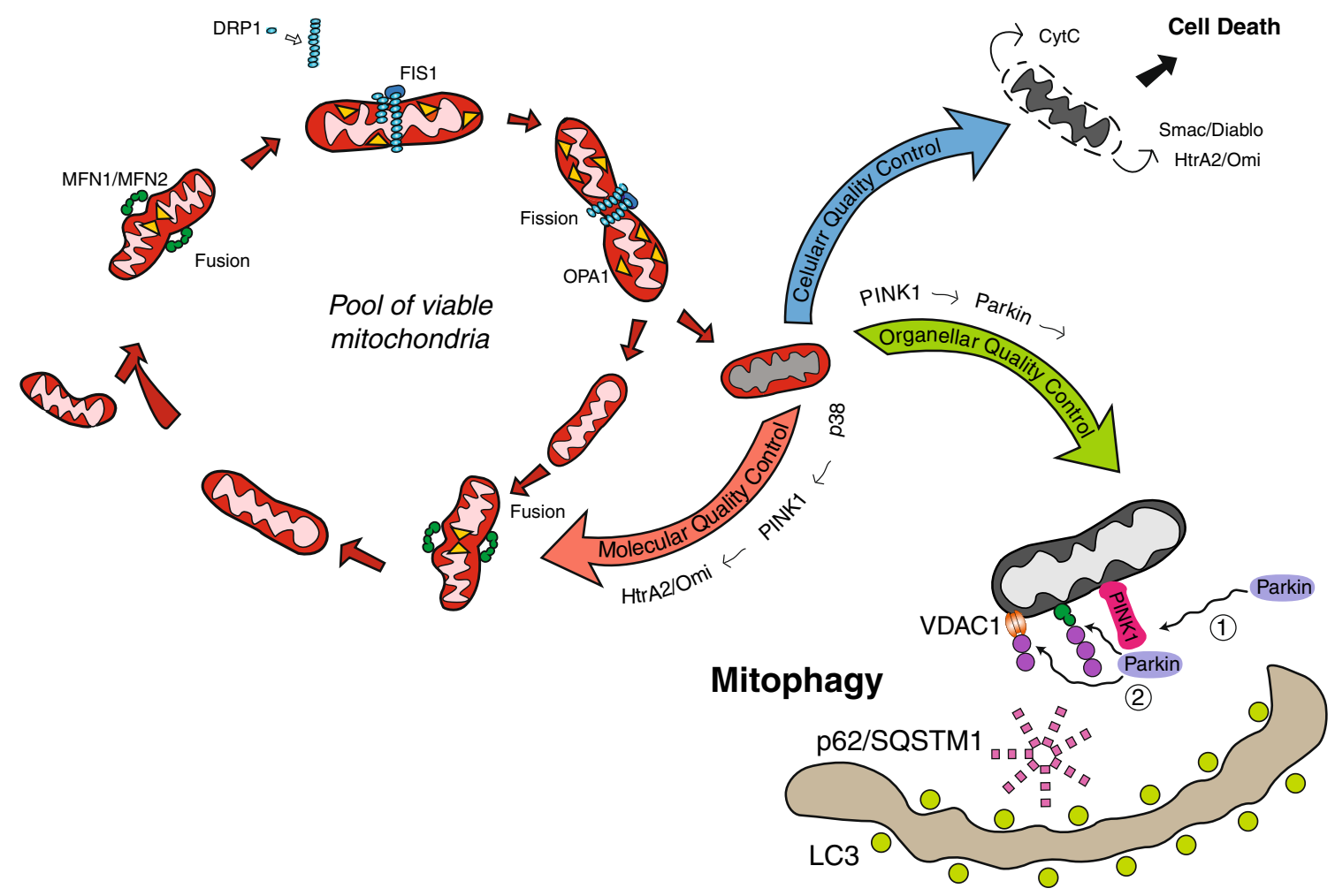

Fig. 2 Dynamics of mitochondrial quality control. Mitochondria are dynamic organelles that are continuously remodelled by fusion and fission events. In this figure, mitochondrial fission is represented as one step of the mitochondrial life cycle involved in the maintenance of a pool of viable mitochondria. In this model, mitochondria can also employ fission to segregate defective daughter mitochondria based on a mechanism that requires OPA1. These defective mitochondria can follow three separate routes. They can be repaired through molecular quality control pathways that possibly involve p38, PINK1 and HtrA2. The defective mitochondria can also be selectively removed through

neurons [4]. Interestingly, mitochondria lacking HtrA2 show increased levels of unfolded components of the ETC [30], indicating that this protease is an important component of the molecular quality control in mitochondria and that disturbances in related quality control mechanisms might underlay parkinsonian neurodegeneration.

In bacteria, the DegS protease is activated by unfolded outer-membrane porins. Studies focusing on the mechanisms of activation of mammalian HtrA2 have shown that this protease is likely to be activated through PINK1dependent phosphorylation. These observations, however, have failed to demonstrate direct phosphorylation of HtrA2 by PINK1 and suggest that PINK1 might instead act as a mitochondrial adaptor for the $\mathrm{p} 38$ stress-activated kinase [31]. Plun-Favreau et al. showed that PINK1 is involved in the phosphorylation of HtrA2; however, this protease is unlikely to be a direct target of the putative kinase activity of PINK1. Taken together, these findings suggest that HtrA2 is possibly a downstream effector of PINK1 in a mitochondrial molecular quality control pathway. autophagy using a mechanism that involves the PINK1-dependent recruitment of the ubiquitin ligase Parkin (1), followed by the ubiquitination of proteins such as VDAC1 and MFN2 (orange and green colours, respectively; purple ubiquitin) (2). Such ubiquitinated proteins might then work as adaptors for p62/SQSTM1 and promote the translocation of defective mitochondria to the autophagosome. Finally, if both molecular and organellar quality control mechanisms fail, mitochondria will ultimately release pro-apoptotic proteins (as indicated on the upper right of the figure), leading to cell death

The emerging picture suggests that PINK1 is a modulator of not only molecular but also organellar quality control in mitochondria. Surprisingly, it was shown that PINK1 is capable of recruiting Parkin, a cytosolic ubiquitin ligase, to damaged mitochondria $[32,33]$ and targeting these organelles for autophagic clearance. The importance of Parkin as a downstream effector of PINK1 has been further confirmed from a series of impressive genetic studies in Drosophila that showed that expression of parkin could rescue the phenotype of pinkl mutant flies [16, 17, 34]. Ubiquitin has been suggested to act as a signal for the selective autophagy of diverse cargos, including protein aggregates, ribosomes, peroxisomes and pathogens in mammalian cells [35]. It seems that, in damaged mitochondria, PINK1 recruits Parkin to catalyse the ubiquitination of proteins close to the outer mitochondrial membrane. Two of the recently identified mitochondrial substrates for Parkin are the voltage-dependent anion channel 1 (VDAC1) [36] and Mitofusin (Mfn) [37]. It remains to be determined if these are specific substrates for Parkin or, upon PINK1- 
dependent mitochondrial recruitment, if Parkin simply "labels" any outer membrane protein with ubiquitin. The mechanism through which ubiquitin targets defective mitochondria for autophagy remains unclear. The mammalian adaptor protein p62/SQSTM1 (sequestosome-1) is an autophagy receptor that can interact with ubiquitin conjugated to a target protein as well as LC3/GABARAP on autophagosomes [35]. It is therefore possible that ubiquitinated proteins on the outer mitochondrial membrane use p62/SQSTM1 as an adaptor to anchor defective mitochondria to the autophagosome. This model is supported by a recent study in which p62/SQSTM1 was found to be recruited to clustered mitochondria and to be essential for their final clearance [36].

Taken together, the findings to date suggest that PINK1 is a master regulator of mitochondrial quality control, at least at two levels; it enhances molecular quality control via HtrA2 and enhances organellar quality control via Parkin. It is worth noting that mutations of all of these molecular components of mitochondrial quality control have been linked to PD, suggesting that alternative hits on this common pathway are possibly important in the development and progression of this disease.

\section{Concluding Remarks}

It is clear that unfolded proteins play an important role in the development of PD. Although clear mechanisms for such proteinopathies are well established when these unfolded proteins accumulate in the cytosol, cell nucleus, endoplasmic reticulum and extracellular space, little is known about any causative role of protein aggregation in mitochondria in PD. Although they have limitations, animal models are beginning to unravel a significant role for this phenomenon in PD when PINK1-mediated mitochondrial quality control is impaired.

The molecular pathogenesis of sporadic PD and the basis for selective dopaminergic neuronal loss remains unknown, and it is unclear if there is a genetic basis for the development of this disease in this subgroup of patients. Recently, several genome-wide association studies (GWAS) have been conducted to evaluate the possible genetic basis for PD in sporadic patients. To date, such approaches have failed to identify any genes associated with mitochondrial dysfunction in evaluated patients. Given that multiple lowfrequency risk variants are below the detection limit of current GWAS methodology (see [38]), it is unclear whether mitochondrial gene mutations play any important role in the aetiology of PD in sporadic patients. With the increasing power of gene sequencing technologies, however, it is likely that, if other mitochondrial mutations in PD patients exist, they will come to light in the near future.
Approximately 40 years ago, US President Richard Nixon declared war on cancer by increasing research funding to improve the understanding of cancer biology. At first glance, cancer and neurodegeneration seem to have little in common. Neurodegeneration can be viewed as resulting from the death of postmitotic neurons, whereas cancer cells show an enhanced resistance to cell death. It is now clear that mitochondria are the master controllers of cell survival and death. Interestingly, mitochondrial PINK1 has been recently shown to be important as a survival mechanism downstream of the PI3K/Akt signalling pathway [39]. This pathway is often overactive in cancer cells, suggesting that PINK1 could have oncogenic potential through the protection of healthy mitochondria in cancer cells. Therefore, we reason that understanding the function of PINK1 might help to fight the war on two fronts, as this knowledge will contribute to the elucidation of the role of mitochondria in both cancer and neurodegeneration.

By increasing our knowledge of the power of mitochondria to control cellular life and death, modern scientists can be compared to the Jedi knights of the fictional Star Wars galaxy, who harnessed the power of midichlorians to fight the 'Dark Side'. We hope that, eventually, the 'Dark Side' will not prevail and that such knowledge will one day translate into cures for devastating diseases such as PD.

Acknowledgments We dedicate this review to Prof. Rita LeviMontalcini.

Open Access This article is distributed under the terms of the Creative Commons Attribution Noncommercial License which permits any noncommercial use, distribution, and reproduction in any medium, provided the original author(s) and source are credited.

\section{References}

1. Gray MW, Burger G, Lang BF (1999) Mitochondrial evolution. Science 283:1476-1481

2. Mattson MP, Gleichmann M, Cheng A (2008) Mitochondria in neuroplasticity and neurological disorders. Neuron 60:748766

3. Jenner P (2003) Oxidative stress in Parkinson's disease. Ann Neurol 53(Suppl 3):S26-S36, discussion S36-S38

4. Martins LM et al (2004) Neuroprotective role of the Reaperrelated serine protease HtrA2/Omi revealed by targeted deletion in mice. Mol Cell Biol 24:9848-9862

5. Youle RJ, Strasser A (2008) The BCL-2 protein family: opposing activities that mediate cell death. Nat Rev Mol Cell Biol 9:47-59

6. Tatton WG et al (2003) Apoptosis in Parkinson's disease: signals for neuronal degradation. Ann Neurol 53(Suppl 3):S61-S70, discussion S70-S72

7. Bolanos JP, Almeida A (2010) The pentose-phosphate pathway in neuronal survival against nitrosative stress. IUBMB Life 62: $14-18$ 
8. MacAskill AF, Kittler JT (2010) Control of mitochondrial transport and localization in neurons. Trends Cell Biol 20:102-112

9. Ly CV, Verstreken P (2006) Mitochondria at the synapse. Neuroscientist 12:291-299

10. Chan CS, Gertler TS, Surmeier DJ (2009) Calcium homeostasis, selective vulnerability and Parkinson's disease. Trends Neurosci 32:249-256

11. Valente EM et al (2004) Hereditary early-onset Parkinson's disease caused by mutations in PINK1. Science 304:1158-1160

12. Strauss KM et al (2005) Loss of function mutations in the gene encoding Omi/HtrA2 in Parkinson's disease. Hum Mol Genet 14:2099-2111

13. Thomas KJ, Cookson MR (2009) The role of PTEN-induced kinase 1 in mitochondrial dysfunction and dynamics. Int $\mathrm{J}$ Biochem Cell Biol 41:2025-2035

14. Beal MF (2010) Parkinson's disease: a model dilemma. Nature 466:S8-S10

15. Kim Y et al (2008) PINK1 controls mitochondrial localization of Parkin through direct phosphorylation. Biochem Biophys Res Commun 377:975-980

16. Clark IE et al (2006) Drosophila pink1 is required for mitochondrial function and interacts genetically with parkin. Nature 441:1162-1166

17. Park J et al (2006) Mitochondrial dysfunction in Drosophila PINK1 mutants is complemented by parkin. Nature 441:1157-1161

18. Kitada $T$ et al (2007) Impaired dopamine release and synaptic plasticity in the striatum of PINK1-deficient mice. Proc Natl Acad Sci USA 104:11441-11446

19. Morais VA et al (2009) Parkinson's disease mutations in PINK1 result in decreased Complex I activity and deficient synaptic function. EMBO Mol Med 1:99-111

20. Gandhi S et al (2009) PINK1-associated Parkinson's disease is caused by neuronal vulnerability to calcium-induced cell death. Mol Cell 33:627-638

21. de Castro IP, Martins LM, Tufi R (2010) Mitochondrial quality control and neurological disease: an emerging connection. Expert Rev Mol Med 12:e12

22. Tatsuta T, Langer T (2008) Quality control of mitochondria: protection against neurodegeneration and ageing. EMBO J $27: 306-314$

23. Broadley SA, Hartl FU (2008) Mitochondrial stress signaling: a pathway unfolds. Trends Cell Biol 18:1-4

24. Twig $G$ et al (2008) Fission and selective fusion govern mitochondrial segregation and elimination by autophagy. EMBO J 27:433-446
25. Faccio L et al (2000) Characterization of a novel human serine protease that has extensive homology to bacterial heat shock endoprotease HtrA and is regulated by kidney ischemia. J Biol Chem 275:2581-2588

26. Li W et al (2002) Structural insights into the pro-apoptotic function of mitochondrial serine protease $\mathrm{HtrA} 2 / \mathrm{Omi}$. Nat Struct Biol 9:436-441

27. Wilken C et al (2004) Crystal structure of the DegS stress sensor: how a PDZ domain recognizes misfolded protein and activates a protease. Cell 117:483-494

28. Martins LM et al (2003) Binding specificity and regulation of the serine protease and PDZ domains of HtrA2/Omi. J Biol Chem 278:49417-49427

29. Ruiz N, Silhavy TJ (2005) Sensing external stress: watchdogs of the Escherichia coli cell envelope. Curr Opin Microbiol 8:122126

30. Moisoi $\mathrm{N}$ et al (2009) Mitochondrial dysfunction triggered by loss of $\mathrm{HtrA} 2$ results in the activation of a brain-specific transcriptional stress response. Cell Death Differ 16:449-464

31. Plun-Favreau $\mathrm{H}$ et al (2007) The mitochondrial protease HtrA2 is regulated by Parkinson's disease-associated kinase PINK1. Nat Cell Biol

32. Narendra D et al (2008) Parkin is recruited selectively to impaired mitochondria and promotes their autophagy. J Cell Biol 183:795803

33. Narendra DP et al (2010) PINK1 is selectively stabilized on impaired mitochondria to activate. Parkin PLoS Biol 8: e1000298

34. Yang $\mathrm{Y}$ et al (2006) Mitochondrial pathology and muscle and dopaminergic neuron degeneration caused by inactivation of Drosophila Pink1 is rescued by Parkin. Proc Natl Acad Sci USA 103:10793-10798

35. Kirkin V et al (2009) A role for ubiquitin in selective autophagy. Mol Cell 34:259-269

36. Geisler S et al (2010) PINK1/Parkin-mediated mitophagy is dependent on VDAC1 and p62/SQSTM1. Nat Cell Biol

37. Ziviani E, Tao RN, Whitworth AJ (2010) Drosophila parkin requires PINK1 for mitochondrial translocation and ubiquitinates mitofusin. Proc Natl Acad Sci USA 107:5018-5023

38. Gandhi S, Wood NW (2010) Genome-wide association studies: the key to unlocking neurodegeneration? Nat Neurosci 13:789794

39. Mei Y et al (2009) FOXO3a-dependent regulation of Pink1 (Park6) mediates survival signaling in response to cytokine deprivation. Proc Natl Acad Sci USA 106:5153-5158 